\title{
Stochastic analysis of a non-local fractional viscoelastic bar forced by Gaussian white noise
}

\author{
G. Alotta \\ Department of Civil, \\ Environmental, Aerospace, \\ Materials Engineering (DICAM) \\ University of Palermo \\ Viale delle Scienze Ed. 8, \\ 90128 Palermo, Italy. \\ Email: gioacchino.alotta@unipa.it
}

\author{
G. Failla \\ Departiment of Civil, \\ Energy, Environment, \\ Materials Engineering (DICEAM) \\ University of Reggio Calabria \\ Via Graziella, Località Feo di Vito, \\ 89124 Reggio Calabria, Italy. \\ Email: giuseppe.failla@unirc.it
}

\author{
F. P. Pinnola \\ Engineering and Architecture Faculty \\ University of Enna "Kore" \\ Viale delle Olimpiadi, \\ 94100 Enna, Italy. \\ Email: francescopaolo.pinnola@unikore.it
}

\begin{abstract}
Recently, a displacement-based non-local bar model has been developed. The model is based on the assumption that non-local forces can be modeled as viscoelastic long-range interactions mutually exerted by non-adjacent bar segments due to their relative motion; the classical local stress resultants are also present in the model. A finite element (FE) formulation with closed-form expressions of the elastic and viscoelastic matrices has also been obtained. Specifically, Caputo's fractional derivative has been used in order to model viscoelastic long-range interaction. The static and quasistatic response has been already investigated. This work investigates the stochastic response of the non-local fractional viscoelastic bar introduced in previous papers, discretized with the FEM, forced by a Gaussian white noise. Since the bar is forced by a Gaussian white noise, dynamical effects cannot be neglected. The system of coupled fractional differential equations ruling the bar motion can be decoupled only by means of the fractional order state variable expansion. It is shown that following this approach Monte Carlo simulation can be performed very efficiently. For simplicity, here the work is limited to the axial response, but can be easily extended to transverse motion.
\end{abstract}

\section{Introduction}

In the last decades, the non-local beam theories have known a great interest. This is due to their ability to capture the mechanical behavior of beam-like micro- and nanodevices [1-4] that avoid computationally expensive (and sometimes prohibitive) molecular simulations [5]; indeed the be- haviour of such micro- and nano-elements can not be reproduced correctly by the classical local continuum approach [6-9]. Moreover this models are also useful for macroscale applications; as an example this is the case of long-fiber reinforced composites, beam with external patches, surface treatments or long adhesive joints in composites where, instead of modeling all components of the system, the coupling between non-adjacent points is taken into account by appropriate non local terms [10-13].

There are several non-local theories available in literature; the most known is for sure the Eringen's integral theory [14, 15], successfully applied to Euler-Bernoulli (EB) model [16] and also to model non-local bi-dimensional elements [17]; however other theories are also effective and have been used to construct non-local beams model based on the kinematic of Euler-Bernoulli (EB), Timoshenko (TM) and higher order beam models: modified couple stress theory [18--20], general strain gradient elasticity theory [8], gradient elasticity theory and integral elasticity theory [21], micropolar elasticity law [9] and also hybrid approaches [22].

In the non-local beam theories listed above, the non-locality is only related to the pure stiffness. However recently a great effort has been dedicated by researchers of the the field to the modeling of non-local damping effects. Indeed, recent studies demonstrate that non-local damping effects at microscale are relevant in application like image acquisition via highspeed atomic force microscopes [23] or frequency measurements of vibrating nanosensors [24]; damping effects have also been observed as a result of humidity and thermal effects [25] or external magnetic forces [26]. As for the non 
Copyright focal damping effects at macroscale, clear examples are the application on beams of external damping patches, adhesive joints, surface treatments or the presence of fibers in composites that produce a coupling between nonadjacent points in terms of viscoelastic forces [12, 13].

In the last years the authors have proposed non-local EB and TM beam models which treats non-local effects as longrange interactions depending on the relative motion of nonadjacent volume elements [27-[30]. In particular non-local forces are considered as volume forces resulting from a relative motion of non-adjacent beam segments; the relative motion is measured by the pure deformation modes of the beams [31, 32] and weighted by a spatial attenuation function. The model is suitable for finite element (FE) implementation and closed form of the FE formulation can be readily found [30]. Both elastic and viscous long-range interactions have been included in this model. Other examples of FE formulation of non-local elasticity can be found in [33, 34].

Recently the non-local TM beam just described has been modified in order to include fractional order viscoelastic long-range interactions [35]. Indeed it is well know that fractional order viscoelasticity is very effective for modeling memory effects of materials [36-42]. However, in the paper [35] only the quasi-static problem has been studied, because when the mass is included the coupled FE equations of motions can not be uncoupled by standard methods of modal analysis. For this reason in this paper the analysis of the FE discretized non-local bar is performed by means of the fractional order state variable expansion [43, 44] that allows to easily decouple the governing equations of motion. With this strategy it is possible to evaluate the solution when the bar is forced either by deterministic or stochastic load. Specifically, in this paper the bar is forced by a Gaussian white noise acceleration impressed at the constrained end. It is shown that results of Monte Carlo simulation perfectly agree with analysis in frequency domain.

\section{Non-local fractional viscoelastic model}

In this section the mechanical model of non-local fractional viscoelastic bar is briefly introduced.

As shown in Fig. 1 the bar has an arbitrary cross section with area $A$, it is referred to an axis $x$ coincident with centroidal axis; the material of the bar is assumed linearly elastic characterized by the Young modulus $E$.

Under the assumptions of small displacements, the kinematics of the bar can be completely described by the following:

$$
\varepsilon(x, t)=\frac{\partial u(x, t)}{\partial x} .
$$

The local axial resultant $N^{(l)}(x, t)$ is

$$
N^{(l)}(x, t)=\int_{A} \sigma_{x}(x, t) d A=\sigma_{x}(x, t) A,
$$

where $\sigma_{x}(x, t)$ is the local stress in the $x$ direction and the second equality holds because for the bar the stress is uniform

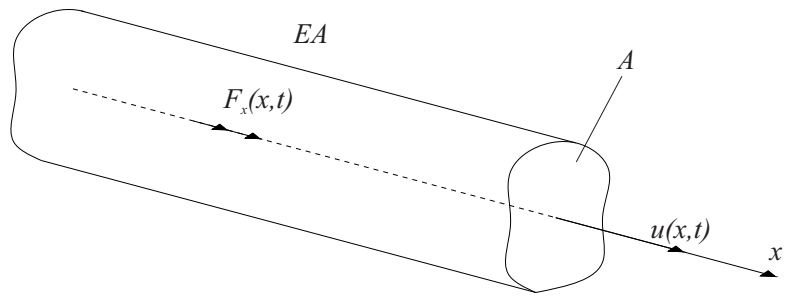

Figure 1. Non local bar.

over the cross section. Local axial resultant is related to the axial strain $\varepsilon_{x}(x, t)$. That is,

$$
N^{(l)}(x, t)=E^{*} A \varepsilon_{x}(x, t)
$$

where $E^{*}=\beta_{1} E$ and $\beta_{1}$ is a dimensionless parameter with values in the range $0 \div 1$, that reduces the amount of local effects [45]. The coefficient $\beta_{1}$ is introduced here as in those non-local theories where the non-local material is thought as a two phase material [46, 47].

\subsection{Long-range forces}

The non-local model is constructed under the assumption that non-adjacent bar segment mutually exert long-range viscoelastic forces due to relative motion. More specifically, consider two nonadjacent bar segment of volume $\Delta V\left(x_{i}\right)$ and $\Delta V\left(\xi_{k}\right)$ located at the positions $x=x_{i}$ and $x=\xi_{k}$ on the bar axis, respectively; they mutually exert long-range axial force as a consequence of their relative motion measured as pure axial deformation [31, 32]. The force are supposed to be selfequilibrated according to the Newton's third law. The longrange forces are written as linearly depending on the product of the two volumes and the attenuation function governing the decay of non-local effects with the relative distance; both purely elastic and fractional viscoelastic forces, modeled by Caputo's fractional derivative, are considered. A mechanical description of the long range interactions is depicted in Fig. 2.

The pure axial deformation $\eta$ is defined as the difference between the displacements of the two bar segments:

$$
\eta\left(x_{i}, \xi_{k}, t\right)=u\left(\xi_{k}, t\right)-u\left(x_{i}, t\right)
$$

The long range axial forces mutually exerted by the two volumes $\Delta V\left(x_{i}\right)$ and $\Delta V\left(\xi_{k}\right)$, due to the axial deformation $\eta$, is given as:

$$
q_{x}\left(x_{i}, \xi_{k}, t\right)=r\left(x_{i}, \xi_{k}, t\right)+d_{x}\left(x_{i}, \xi_{k}, t\right)
$$

$$
r_{x}\left(x_{i}, \xi_{k}, t\right)=g_{x}\left(x_{i}, \xi_{k}\right) \eta\left(x_{i}, \xi_{k}, t\right) \Delta V\left(x_{i}\right) \Delta V\left(\xi_{k}\right)
$$

$$
d_{x}\left(x_{i}, \xi_{k}, t\right)=\tilde{g}_{x}\left(x_{i}, \xi_{k}\right)\left(D^{\alpha} \eta\right)\left(x_{i}, \xi_{k}, t\right) \Delta V\left(x_{i}\right) \Delta V\left(\xi_{k}\right)
$$



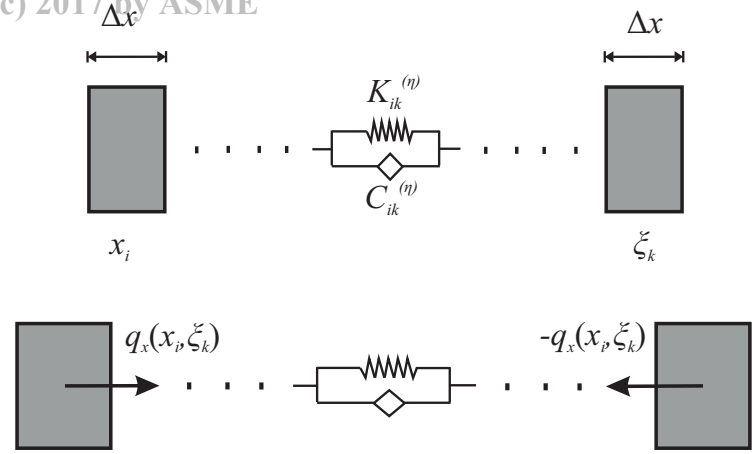

$\eta>0$

Figure 2. Pure axial deformation $\eta$ and long range forces

where $g_{x}$ and $\tilde{g}_{x}$ are the attenuation function of the long range elastic and fractional viscoelastic interactions, respectively. Typically, these functions are chosen as Gaussian, exponential or power law [13, 30]. In Eq. $[5 \mathrm{c})\left(D^{\alpha} \cdot\right)(t)$ is the time Caputo's fractional derivative of order $\alpha$ defined as:

$$
\left(D^{\alpha} f\right)(t)=\frac{1}{\Gamma(1-\alpha)} \int_{0}^{t}(t-\tau)^{-\alpha} \dot{f}(\tau) d \tau
$$

being $\alpha$ a real number, $\Gamma(\cdot)$ the Euler gamma function [36] and dot means differentiation with respect to time; in viscoelasticity the order of derivation $\alpha$ assumes values in the range $0 \div 1$ [36, 37]. The Caputo fractional derivative naturally arises when creep and relaxation behavior are well fitted by power law functions of real order in linear viscoelasticity. Indeed, by arranging the power law relaxation function as:

$$
G(t)=\frac{C_{\alpha} t^{-\alpha}}{\Gamma(1-\alpha)}
$$

and introducing it in the Boltzmann superposition principle, the following constitutive equation is obtained:

$$
F(t)=C_{\alpha}\left(D^{\alpha} u\right)(t)
$$

where $F(t)$ is the applied force and $u(t)$ is the correspondent displacement. By considering a power law creep function, the application of Boltzmann superposition principle leads directly to inverse of Eq. (8):

$$
u(t)=\frac{1}{C_{\alpha}}\left(I^{\alpha} F\right)(t)
$$

being $\left(I^{\alpha} \cdot\right)$ the Riemann-Liouville fractional integral.

The fractional order element is usually represented as a rhombus (see Fig. 2) and is labeled springpot. From Fig. 2 it is clear that, while local forces are considered as purely elastic, the long range forces are modeled as a fractional KelvinVoigt viscoelastic model [38].

More informations about fractional calculus and fractional order viscoelasticity can be found in [36, 48].

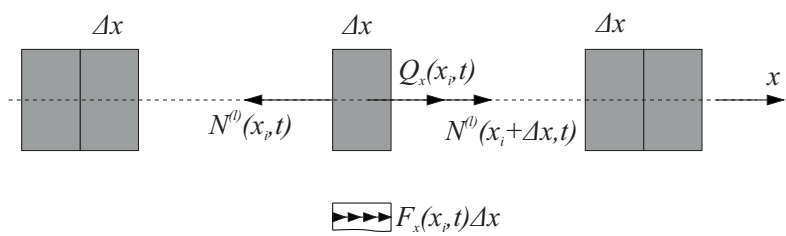

Figure 3. Axial equilibrium of a bar segment; positive sign conventions are reported.

\subsection{Non-local bar equation of motion}

Let us divide the bar in $N$ segments of length $\Delta x$ and consider the bar segment of $\Delta V\left(x_{i}\right)=A \Delta x$ at the location $x=x_{i}=i \Delta x$, with $i=0,1, \ldots, N$; the equation of motion of this bar segment is

$$
\begin{array}{r}
N^{(l)}\left(x_{i}+\Delta x\right)-N^{(l)}\left(x_{i}\right)+Q_{x}\left(x_{i}, t\right)+F_{x}\left(x_{i}, t\right) \Delta x- \\
m\left(x_{i}\right) \ddot{u}\left(x_{i}, t\right) \Delta x=0,
\end{array}
$$

where $Q_{x}\left(x_{i}, t\right)$ is the resultant of non-local forces on the beam segment at hand, $F_{x}\left(x_{i}, t\right)$ is the external axial force per unit-length, $m(x)=\rho(x) A$ being $\rho(x)$ the mass per unit volume. The term $Q_{x}\left(x_{i}, t\right)$ is the resultant of the non-local forces coming form the relative displacements between the bar segment at location $x_{i}$ and all other bar segments. That is,

$$
Q_{x}\left(x_{i}, t\right)=\sum_{k=0, k \neq i}^{N-1} q_{x}\left(x_{i}, \xi_{k}, t\right)
$$

By considering Eq. (5a) for $q_{x}$, dividing Eq. (10) by $\Delta x$ and performing the limit for $\Delta x \rightarrow 0$, the continuous counterpart of Eq. (10) is obtained:

$$
\begin{gathered}
E^{*} A \frac{\partial^{2} u(x, t)}{\partial x^{2}}+F_{x}(x, t)+A^{2} \int_{0}^{L}\left\{g_{x}(x, \xi) \eta(x, \xi, t)+\right. \\
\left.\tilde{g}_{x}(x, \xi)\left(D^{\alpha} \eta\right)(x, \xi, t)\right\} d \xi=m(x) \ddot{u}(x, t) .
\end{gathered}
$$

Regards the boundary conditions (BCs), it can be easily seen that the $\mathrm{BCs}$ of the classical local theory still hold since in the equilibrium equation at the bar ends, the long-range resultants are infinitesimal of higher order with respect to the local resultants [49]. Then, since time-independent kinematic $\mathrm{BCs}$ are considered, they are given as:

$$
\left.E^{*} A \frac{\partial u(x, t)}{\partial x}\right|_{x=x_{i}}=\mp N_{i}(t), \quad \text { or } \quad u\left(x_{i}, t\right)=u_{i},
$$

where $N_{i}$ and $u_{i}$ are forces and displacements at the beam ends, that is $x_{0}=0$ and $x_{N}=L$. 


\section{Copyright $2.3^{20}$ Finite element formulation}

The displacement based non-local model of the bar is suitable for implementation in FE method. To this purpose, let us divide the bar in $n$ finite elements of the same length $l$, such that $n l=L$, being $L$ the length of the bar. The points shared by adjacent bar elements are the nodes; the generic $i-t h$ element has two nodes located at $x=\hat{x}_{i}=(i-1) l$ and $x=\hat{x}_{i+1}=i l$. The displacement field within the element is approximated by means of standard linear shape functions as follows:

$$
\begin{gathered}
u_{i}(x, t)=\boldsymbol{N}_{i}(x) \boldsymbol{d}_{i}(t), \quad i=1,2, \ldots, n, \\
\boldsymbol{d}_{i}(t)=\left[u_{(i) 1}(t) u_{(i) 2}(t)\right],
\end{gathered}
$$

where $u_{(i) 1}(t)$ and $u_{(i) 2}(t)$ are the axial displacements of the two nodes of the $i-t h$ element and $N_{i}(x)$ is the shape functions vector of the $i-t h$ element, that is

$$
\boldsymbol{N}_{i}(x)=\left[\begin{array}{ll}
\frac{\hat{x}_{i+1}-x}{l} & \frac{x-\hat{x}_{i}}{l}
\end{array}\right]
$$

Next, being $\boldsymbol{d}^{T}(t)=\left[u_{1}(t) u_{2}(t) \ldots u_{n+1}(t)\right]^{T}$ the vector collecting the displacements of all nodes, the nodal displacements of the $i-t h$ element are written in the form:

$$
\boldsymbol{d}_{i}(x)=\boldsymbol{C}_{i} \boldsymbol{d}(t)
$$

where $C_{i}$ is the connectivity matrix of the $i-t h$ element. Following a standard Galerkin approach, the dynamic equilibrium equation of the discretized bar is

$$
\boldsymbol{M} \ddot{\boldsymbol{d}}(t)+\boldsymbol{C}^{(n l)}\left(D^{\alpha} \boldsymbol{d}\right)(t)+\boldsymbol{K} \boldsymbol{d}(t)=\boldsymbol{F}(t),
$$

being $\boldsymbol{M}$ the consistent mass matrix [50], $\boldsymbol{C}^{(n l)}$ the matrix of fractional viscoelastic long range interactions, $\boldsymbol{K}$ the stiffness matrix and $\boldsymbol{F}(t)$ the vector of nodal forces. The stiffness matrix is obtained as

$$
\boldsymbol{K}=\boldsymbol{K}^{(l)}+\boldsymbol{K}^{(n l)}=\sum_{i=1}^{n} \boldsymbol{K}_{i}^{(l)}+\sum_{i=1}^{n} \boldsymbol{K}_{i}^{(n l)}
$$

where $\boldsymbol{K}^{(l)}$ and $\boldsymbol{K}^{(n l)}$ are the local and non-local stiffness contribution to the stiffness, respectively. The local stiffness matrix of the $i-t h$ element is

$$
\boldsymbol{K}_{i}^{(l)}=\int_{\hat{x}_{i}}^{\hat{x}_{i+1}}\left[\boldsymbol{B}_{i}(x) \boldsymbol{C}_{i}\right]^{T} E^{*} A \boldsymbol{B}_{i}(x) \boldsymbol{C}_{i} d x
$$

where $\boldsymbol{B}_{i}(x)$ is the vector collecting the spatial derivative of the shape functions. That is,

$$
\boldsymbol{B}_{i}(x)=\left[\begin{array}{ll}
-\frac{1}{l} & \frac{1}{l}
\end{array}\right]
$$

The non-local stiffness matrix of the $i-t h$ element $\boldsymbol{K}_{i}^{(n l)}$ is evaluated as

$$
\boldsymbol{K}_{i}^{(n l)}=\sum_{j=1}^{n} \boldsymbol{K}_{i j}^{(n l)}
$$

with

$$
\begin{array}{r}
\boldsymbol{K}_{i j}^{(n l)}=\frac{A^{2}}{2} \int_{\hat{x}_{i}}^{\hat{x}_{i+1}} \int_{\hat{x}_{j}}^{\hat{x}_{j+1}}\left[\boldsymbol{N}_{j}(\boldsymbol{\xi}) C_{j}-\boldsymbol{N}_{i}(x) C_{i}\right]^{T} g_{x}(x, \boldsymbol{\xi}) \\
{\left[\boldsymbol{N}_{j}(\boldsymbol{\xi}) C_{j}-\boldsymbol{N}_{i}(x) C_{i}\right] d x d \xi .}
\end{array}
$$

It is to emphasized that the matrix $\boldsymbol{C}^{(n l)}$ has the same mathematical form of the non-local stiffness matrix $\boldsymbol{K}^{(n l)}$; the only difference is that in $\boldsymbol{C}^{(n l)} g_{x}(x, \xi)$ has been replaced by $\tilde{g}_{x}(x, \xi)$. Finally, the vector $\boldsymbol{F}(t)$ is given as:

$$
\boldsymbol{F}(t)=\sum_{i=1}^{n} \boldsymbol{F}_{i}(t)
$$

where

$$
\begin{array}{r}
\boldsymbol{F}_{i}(t)=\int_{V_{i}}\left[\boldsymbol{N}_{i}(x) \boldsymbol{C}_{i}\right] F_{x}(x, t) d V_{i}(x)+\left[\boldsymbol{N}_{1}(0) \boldsymbol{C}_{i}\right]^{T} N_{1}(t)+ \\
{\left[\boldsymbol{N}_{n+1}(L) \boldsymbol{C}_{n+1}\right]^{T} N_{n+1}(t)}
\end{array}
$$

\section{Stochastic response of non-local bar}

In the previous section the finite element formulation of fractional viscoelastic non-local bar has been presented. Now, such problem is considered for the case in which the external load vector in Eq. (17) is composed by stochastic actions. In order to obtain the numerical and the analytical solution of the problem at hand, some specific mathematical tools of the fractional calculus will be used.

\subsection{Problem formulation}

Consider that the coupled fractional differential equations in Eq. (17) is forced by a stochastic input at the constrained end. In particular, the cantilever rod is forced by a Gaussian white noise acceleration impressed at the constrained end in axial direction. Such white noise with zero mean is denoted by $W(t)$. In this way the external force vector is $\boldsymbol{F}(t)=-\boldsymbol{M} \boldsymbol{u} W(t)$, being $\boldsymbol{u}^{T}=$ $[1,1, \ldots, 1]$, and then the vector of response function $\boldsymbol{d}^{T}(t)=$ $\left[U_{1}(t), U_{2}(t), \ldots, U_{n+1}(t)\right]$ is a set of stochastic processes too. In order to distinguish the deterministic functions from the stochastic ones, the response function and the forced function will be denoted by capital letters. Then, in the stochastic case the Eq. 17) becomes

$$
\boldsymbol{M} \ddot{\boldsymbol{d}}(t)+\boldsymbol{C}^{(n l)}\left(D^{\alpha} \boldsymbol{d}\right)(t)+\boldsymbol{K} \boldsymbol{d}(t)=\boldsymbol{v} W(t),
$$


Copyright being $\boldsymbol{v} \stackrel{\text { by }}{=} \boldsymbol{M} \boldsymbol{u}$. The power spectral density (PSD) of the Gaussian white noise $W(t)$ is constant, and the correlation function $(\mathrm{CF})$ is a Dirac delta function. That is,

$$
S_{W}(\omega)=S_{0}, \quad R_{W}(\tau)=2 \pi \delta(\tau),
$$

where $\delta(\tau)$ is the Dirac delta function, $S_{W}(\omega)$ and $R_{W}(\tau)$ denote the PSD and CF of white noise, respectively.

The system in Eq. (25) is linear, since the fractional derivative is a linear operator, and the set of nodal displacements $\boldsymbol{d}(t)$ is composed by Gaussian processes. Therefore, each response process can be characterized at steady state by two deterministic function. That is, the PSD, and the CF. It has been shown that for the fractional differential equations forced by Gaussian white noise the stationary PSD can be evaluated in closed form but the CF can be obtained just in approximated way by the discrete inverse Fourier transform or by using the complex spectral moments [51].

\subsection{Stationary response characterization}

The stationary analysis in frequency domain in terms of PSD determination is particularly useful for the evaluation of the stationary statistics of the response. This aim can be pursued with the direct evaluation of the PSD of the responses from the coupled differential equations in Eq. 25. In fact, the Fourier transform of such equation yields

$$
\left[-\omega^{2} \boldsymbol{M}+(i \omega)^{\alpha} \boldsymbol{C}^{(n l)}+\boldsymbol{K}\right] \boldsymbol{d}_{\mathscr{F}}(\omega, T)=\boldsymbol{v} W_{\mathscr{F}}(\omega, T)
$$

where the $i=\sqrt{-1}$ is the imaginary unit, $\boldsymbol{d}_{\mathscr{F}}(\omega, T)$ contains the truncated Fourier transform of the response processes, and $W_{F}(\omega, T)$ denotes the Fourier transform of the Gaussian white noises truncated at time $\mathrm{T}$ in the frequency domain $\omega$. Observe that the power law $(i \omega)^{\alpha}$, related to the fractional order terms, introduces in the system a double effect. In fact, such power law term contains an effective stiffness (related to the $\Re\left[(i \omega)^{\alpha}\right]$ ) and an effective damping (proportional to the $\left.\mathfrak{I}\left[(i \omega)^{\alpha}\right]\right)$. By Eq. 25 the response in the frequency domain is

$$
\begin{aligned}
\boldsymbol{d}_{\mathscr{F}}(\omega, T) & =\left[-\omega^{2} \boldsymbol{M}+(i \omega)^{\alpha} \boldsymbol{C}^{(n l)}+\boldsymbol{K}\right]^{-1} \boldsymbol{v} W_{\mathscr{F}}(\omega, T) \\
& =\boldsymbol{H}(\omega) \boldsymbol{v} W_{\mathscr{F}}(\omega, T)
\end{aligned}
$$

where $\boldsymbol{H}(\omega)$ contains the transfer functions.

In order to fully characterize the stationary response in terms of displacements $U_{j}(t)$ for $j=1,2, \ldots, n+1$, the evaluation of the PSD and all the cross PSD of each element of the vector $\boldsymbol{d}(t)$ is needed. In this regard, consider the PSD matrix defined as

$$
\begin{aligned}
\boldsymbol{S}_{d}(\omega) & =\lim _{T \rightarrow \infty} \frac{1}{2 \pi T} \mathrm{E}\left[\boldsymbol{H}^{*}(\omega) \boldsymbol{v} W_{\mathscr{F}}^{*}(\omega, T) W_{\mathscr{F}}(\omega, T) \boldsymbol{v}^{T} \boldsymbol{H}^{T}(\omega)\right] \\
& =\boldsymbol{H}^{*}(\omega) \boldsymbol{v} \lim _{T \rightarrow \infty} \frac{1}{2 \pi T} \mathrm{E}\left[W_{\mathscr{F}}^{*}(\omega, T) W_{\mathscr{F}}(\omega, T)\right] \boldsymbol{v}^{T} \boldsymbol{H}^{T}(\omega) \\
& =\boldsymbol{H}^{*}(\omega) \boldsymbol{v} S_{0} \boldsymbol{v}^{T} \boldsymbol{H}^{T}(\omega),
\end{aligned}
$$

where $\mathrm{E}[\cdot]$ is the expectation value and the apex $*$ denotes the complex conjugate. Consequently, the matrix $S_{d}(\omega)$ is

$$
\boldsymbol{S}_{d}(\omega)=\left[\begin{array}{cccc}
S_{U_{1}}(\omega) & S_{U_{1} U_{2}}(\omega) & \ldots & S_{U_{1} U_{n+1}} \\
S_{U_{2} U_{1}}(\omega) & S_{U_{2}}(\omega) & \ldots & S_{U_{2} U_{n+1}} \\
\vdots & \vdots & \ddots & \vdots \\
S_{U_{n+1} U_{1}}(\omega) & S_{U_{n+1} U_{2}}(\omega) & \ldots & S_{U_{n+1}}
\end{array}\right]
$$

and each term represents the power spectral density function of the output processes and their cross counterparts. In particular, the diagonal terms are the PSDs, whereas the other terms are the cross PSDs. Moreover, being $S_{U_{j} U_{k}}(\omega)=$ $S_{U_{k} U_{j}}(\omega)$ the matrix is Hermitian. All terms of $S_{d}(\omega)$ can be evaluated from Eq. 29] and they are related to the autocorrelation function $(\mathrm{CF})$ and the cross-correlation function (CCF) of the output processes by the Wiener-Khinchin theorem. That is,

$$
\begin{aligned}
S_{U_{j} U_{k}}(\omega) & =\frac{1}{2 \pi} \int_{-\infty}^{\infty} R_{U_{j} U_{k}}(\tau) \exp (i \omega \tau) d \tau \\
R_{U_{j} U_{k}}(\tau) & =\int_{-\infty}^{\infty} S_{U_{j} U_{k}}(\omega) \exp (-i \omega \tau) d \omega
\end{aligned}
$$

where $R_{U_{j} U_{k}}(\tau)$ represents the CCF of the processes $U_{j}(t)$ and $U_{k}(t)$. Observe that from Eq.s 31 is not possible to obtain the CCFs in analytical form because the second integral can be solved just in numerical way when fractional powers of order $\alpha$ of $i \omega$ appear in the PSDs and in the CPSDs [51]. By Eqs. 31 the stationary variances and covariances can be obtained as

$$
\sigma_{U_{j} U_{k}}=R_{U_{j} U_{k}}(0)=\int_{-\infty}^{\infty} S_{U_{j} U_{k}}(\omega) d \omega
$$

such kind of integrals cannot be solved in analytical form and the variances and the cross-variances have to be evaluated in numerical way. Furthermore, the covariance matrix $\boldsymbol{\Sigma}_{d}$ can be evaluated just by numerical integration of each integral of $S_{U_{j} U_{k}}(\omega)$.

The numerical solution in terms of variances and crossvariances obtained with the aid of the Eq. (32) can be used as benchmark for the method described in the next section in which the stationary and non-stationary solution of the problem at hand are found with the direct integration of the equations of motion. Moreover, with the present frequency domain approach the influence of the fractional order derivative on the PSD of the responses can be readily investigated. 


\section{Copyright $\mathbf{3 . 3 ^ { 2 0 }}$ Time domain solution}

For the evaluation of the statistics of the response processes the Monte Carlo method represents a valuable tool. In the first step the generation of a certain number of samples of the stochastic input is needed. The second step is the integration of the equations of motion. The direct numerical integration of the set of fractional differential equations in Eq. 25] is a cumbersome computational problem because each fractional differential equation is coupled with the others and the classical modal transformation is useless in the general case. For this reason a proper fractional state variable expansion is used in conjunction with a complex modal transformation in order to decouple the system in Eq. 25.

Consider the case in which the fractional order $\alpha$ is rational, under this assumptions it is possible to represent the generic fractional order as irreducible fractions of two integer values $\alpha=a / b$, where $a, b \in \mathbb{N}$. Thus, the system in Eq. 25] can be rewritten as the following sequential linear differential equations of fractional orders:

$$
\sum_{j=1}^{2 b} \boldsymbol{C}_{j}\left(D^{j / b} \boldsymbol{d}\right)(t)+\boldsymbol{K} \boldsymbol{d}(t)=\boldsymbol{v} W(t),
$$

where the involved matrices in the summation are $\boldsymbol{C}_{a}=\boldsymbol{C}^{(n l)}$, $\boldsymbol{C}_{2 b}=\boldsymbol{M}$ and $\boldsymbol{C}_{j}=\mathbf{0}, \forall j: j \in(1, a]$ and $[a, 2 b-1)$. Introducing the vector of state variables

$$
z^{T}(t)=\left[\boldsymbol{d}^{T}(t)\left(D^{1 / b} \boldsymbol{d}^{T}\right)(t) \ldots\left(D^{(2 b-1) / b} \boldsymbol{d}^{T}\right)(t)\right]
$$

and appending to Eq. 33 the $2 b-1$ identities

$$
\begin{array}{r}
\sum_{j=1}^{2 b-k} \boldsymbol{C}_{j+k}\left(D^{1 / b} D^{(j-1) / b} \boldsymbol{d}\right)(t)=\sum_{j=1}^{2 b-k} \boldsymbol{C}_{j+k}\left(D^{j / b} \boldsymbol{d}\right)(t), \\
k=1,2, \ldots, 2 b-1,
\end{array}
$$

then a set of $(n+1) \times 2 b$ coupled differential equations is readily cast in the form

$$
\boldsymbol{A}\left(D^{1 / b} \boldsymbol{z}\right)(t)+\boldsymbol{B} \boldsymbol{z}(t)=\boldsymbol{g}(t)
$$

where $\boldsymbol{g}^{T}(t)=W(t)\left[\begin{array}{lll}\boldsymbol{v}^{T} & \mathbf{0} \ldots & \mathbf{0}\end{array}\right]$, the involved matrices are symmetric and defined as

$$
\boldsymbol{A}=\left[\begin{array}{ccccc}
\boldsymbol{C}_{1} & \boldsymbol{C}_{2} & \ldots & \boldsymbol{C}_{2 b-1} & \boldsymbol{C}_{2 b} \\
\boldsymbol{C}_{2} & \boldsymbol{C}_{3} & \ldots & \boldsymbol{C}_{2 b} & \mathbf{0} \\
\vdots & \vdots & \ddots & \vdots & \vdots \\
\boldsymbol{C}_{2 b-1} & \boldsymbol{C}_{2 b} & \ldots & \mathbf{0} & \mathbf{0} \\
\boldsymbol{C}_{2 b} & \mathbf{0} & \ldots & \mathbf{0} & \mathbf{0}
\end{array}\right]
$$

$$
\boldsymbol{B}=\left[\begin{array}{ccccc}
\boldsymbol{K} & \mathbf{0} & \ldots & \mathbf{0} & \mathbf{0} \\
\mathbf{0} & -\boldsymbol{C}_{2} & \ldots & -\boldsymbol{C}_{2 b-1} & -\boldsymbol{C}_{2 b} \\
\vdots & \vdots & \ddots & \vdots & \vdots \\
\mathbf{0} & -\boldsymbol{C}_{2 b-1} & \cdots & \mathbf{0} & \mathbf{0} \\
\mathbf{0} & -\boldsymbol{C}_{2 b} & \cdots & \mathbf{0} & \mathbf{0}
\end{array}\right]
$$

Next, it is possible to decompose $z(t)$ in the orthogonal basis of the eigenvectors of $\boldsymbol{A}$ and $\boldsymbol{B}$. Specifically, consider the equations

$$
\boldsymbol{\Psi}^{T} \boldsymbol{A} \boldsymbol{\Psi}=\boldsymbol{U}_{\mathrm{d}}, \quad \boldsymbol{\Psi}^{T} \boldsymbol{B} \boldsymbol{\Psi}=\boldsymbol{V}_{\mathrm{d}}
$$

where $\boldsymbol{U}_{\mathbf{d}}$ and $\boldsymbol{V}_{\mathbf{d}}$ are diagonal matrices (subscript $\mathrm{d}$ stands for diagonal), whereas $\boldsymbol{\Psi}$ contains the eigenvectors of the matrix $\boldsymbol{D}=\boldsymbol{A}^{-1} \boldsymbol{B}$. Further, making the complex modal transformation

$$
\boldsymbol{z}(t)=\boldsymbol{\Psi} \boldsymbol{y}(t),
$$

a new set of decoupled fractional differential equation is derived in the form

$$
\boldsymbol{U}_{\mathbf{d}}\left(D^{1 / b} \boldsymbol{y}\right)(t)+\boldsymbol{V}_{\mathbf{d}} \boldsymbol{y}(t)=\boldsymbol{\mu}(t)
$$

being $\boldsymbol{\mu}(t)=\boldsymbol{\Phi}^{T} \boldsymbol{g}(t)$. Clearly, once the decoupled set is found, the fractional differential equations can be readily solved. It can be seen that the state variable problem in Eq. (41) has a greater number of involved variables with respect to the problem cast in terms of displacements in Eq. (25) of the nodal analysis. However, this apparent increase of the computational burden is balanced by the fact that the maximum involved order in the state variables domain is smaller than the maximum order in the nodal analysis. Further, the system in Eq. (25) is a set of coupled in the general case, vis-a-vis the system in Eq. (25) leads readily to the set of uncoupled equations in Eq. 411. From Eq. (41) each decoupled differential equation in the complex modal space is of the type

$$
u_{j}\left(D^{1 / b} y_{j}\right)(t)+v_{j} y_{j}(t)=\mu_{j}(t), \quad j=1,2, \ldots, 2 b,
$$

where $u_{j}$ and $v_{j}$ are the $\mathrm{j}$-th elements of the diagonal matrices $\boldsymbol{U}_{\mathrm{d}}$ and $\boldsymbol{V}_{\mathrm{d}}$, respectively. Each equation represents the equation of motion of a fractional Kelvin-Voigt model with complex coefficients [36, 37]. The impulse response function of the Eq. (42) is

$$
h_{j}(t)=\frac{\sqrt[b]{t}}{t v_{j}} \sum_{k=0}^{\infty} \frac{\left(-v_{j} / u_{j} \sqrt[b]{t}\right)^{k}}{\Gamma(k / b+1 / b)}
$$

where the summation is a Mittag-Leffler function [36]. Clearly, the solution in terms of the modal displacements 


$$
\tilde{g}(x, \xi)=\frac{\tilde{C}}{h^{2}} \exp \left(\frac{|x-\xi|}{\tilde{\lambda}}\right)
$$

$$
y_{j}(t)=\int_{0}^{t} h_{j}(t-\bar{t}) \mu_{j}(\bar{t}) d \bar{t},
$$

and the first $2 b$-terms of the vector $z(t)$ contain the solution in terms of displacements response. In particular, by virtue of the modal transformation in Eq. (40), each displacement is

$$
\begin{array}{r}
U_{l}(t)=z_{l}(t)=\sum_{j=1}^{2 b(n+1)} \sum_{k=1}^{n+1} \psi_{l, j} \psi_{j, k} \int_{0}^{t} h_{j}(t-\bar{t}) W(\bar{t}) d \bar{t} \\
l=1,2, \ldots, n+1
\end{array}
$$

$\psi_{j, l}$ represents the term in the $j$-th row and the $l$-th column of the matrix of the complex eigenvectors $\boldsymbol{\Psi}$. The velocity processes are

$$
\begin{array}{r}
\dot{U}_{l}(t)=z_{l}(t)=\sum_{j=1}^{2 b(n+1)} \sum_{k=1}^{n+1} \psi_{l, j} \psi_{j, k} \int_{0}^{t} h_{j}(t-\bar{t}) W(\bar{t}) d \bar{t} \\
l=n+2, n+3, \ldots, 2(n+1)
\end{array}
$$

Eq.s (45) and (46) can be used for the Monte Carlo simulation. In particular, once a set of sample of Gaussian white noise are generated the response in terms of displacement and velocity processes can be evaluated. After such evaluation the stationary and non stationary variances can be calculated.

The described state variable analysis and the Monte Carlo method is used in the next section to investigate the influence of the fractional order on the statistics of stationary and non-stationary response.

\section{Numerical applications}

The state variable expansion described in the previous section is used now to evaluate the statistics of the stochastic response of the non-local bar forced by Gaussian white noise. In particular, the cantilever rod is forced by Gaussian white noise acceleration impressed at the fixed end in axial direction. Such white noise is characterized by unitary PSD, $S_{0}=1$. The mechanical element is an epoxy micro beam with longitudinal length $L=300 \mu \mathrm{m}$. The bar has a constant cross section with dimensions $b=30 \mu \mathrm{m}$ and $h=15 \mu \mathrm{m}$. Considering that the material is an epoxy resin the elastic modulus is $E=1.4 G P a$, whereas the density is $\rho=1000 \mathrm{Kg} / \mathrm{m}^{3}$. As for the attenuation functions, typical exponential functions have been selected [30, 35]. That is,

$$
g(x, \xi)=\frac{C}{h^{2}} \exp \left(\frac{|x-\xi|}{\lambda}\right)
$$

where $\lambda=\tilde{\lambda}=30 \mu \mathrm{m}, C=10^{22} \mathrm{Nm}^{-6}$ and $\tilde{C}=10^{21} \mathrm{Nm}^{-6}$. For the finite element formulation the bar is divided in forty parts and then $n=40$ nodes are considered. In order to show the effect of the fractional order in the stochastic response of the bar, two different cases are considered. In the first case the chosen fractional order is $\alpha=1 / 4$, whereas in the second case the fractional order is $\alpha=3 / 4$. Such choice aims to show the differences between the response in the case in which the elastic phase is predominant (elasto-viscous (EV) case $\alpha=1 / 4$ ) and the response when the damping effect prevails (visco-elastic (VE) case $\alpha=3 / 4$ ). In both cases the matrices of the coefficients $\boldsymbol{C}_{n l}$ are the same. In this manner only the influence of the fractional order is considered.

For both cases the value $b$ in Eq. (33) that allows to rewrite the equation of motion in the state variables domain is $b=4$ and the number of state variables is $2 b=8$.

By performing the state variable expansion and the complex modal transformation, the Eq. (45) gives each nodal displacements $U_{j}(t)$ for any generic sample of the Gaussian white noise $W(t)$. Such samples have been simulated by the harmonic superposition method by Shinozuka and Deodatis [52]. The number of generated sample for the Monte Carlo simulation is $N=2000$. The time step chosen to generate the samples of $W(t)$ and for the numerical integration of Eq. (45) and the total duration of the simulation $t_{f}$ have been calibrated in such a way that the stationary condition are reached. For this reason in the case $\alpha=1 / 4$ the time step is $\Delta t=10^{-8} s$ and $t_{f}=10^{-5} s$, while in the case $\alpha=3 / 4$ $\Delta t=10^{-6} s$ and $t_{f}=5 \times 10^{-4} s$. After the generation of the input and the numerical integration of the Eq. (45) the statistics can be evaluated at the different nodes. In particular, the considered nodes are the middle-bar node and the free end one.

The stationary and non-stationary variance of the two considered displacements for $\alpha=1 / 4$ are reported in Figure 4, while the results for $\alpha=3 / 4$ are reported in Figure 6. These results are compared with the stationary variances obtained by numerical integration of the PSD functions of the Eq. 29] and depicted in dotted line in Figure 4 for the EV case and in Figure 6 for the VE case.

The PSD of the two considered displacements are reported in Figure 5 for the EV case and in Figure 7 for the VE case.

Comparing the stationary variances and the PSD functions of both EV and VE case some useful consequences can be drawn. In particular, the increasing of the fractional order leads to a reduction of the PSD amplitude. This is related to the increment of damping effect in the frequency domain, in fact when $\alpha$ grows, then the imaginary part of the power law term $(i \omega)^{\alpha}$ in Eq. (28) increases. Such reduction can be observed comparing the PSDs in Figure 5 and 7 and considering the peaks for $\alpha=1 / 4$ in Fig. 5 that do not exist in the other case depicted in Fig. 7. Obviously, it implies that when the fractional order increases the stationary variance 




Figure 4. Displacement variance at $x=L$ (black) and $x=L / 2$ (gray) for EV case $\alpha=1 / 4$.

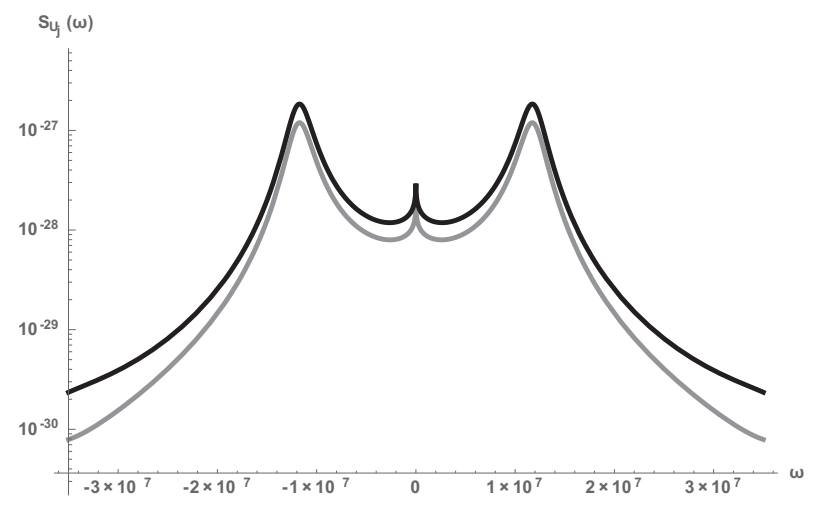

Figure 5. PSD of axial displacement at $x=L$ (black) and $x=L / 2$ (gray) for EV case $\alpha=1 / 4$.

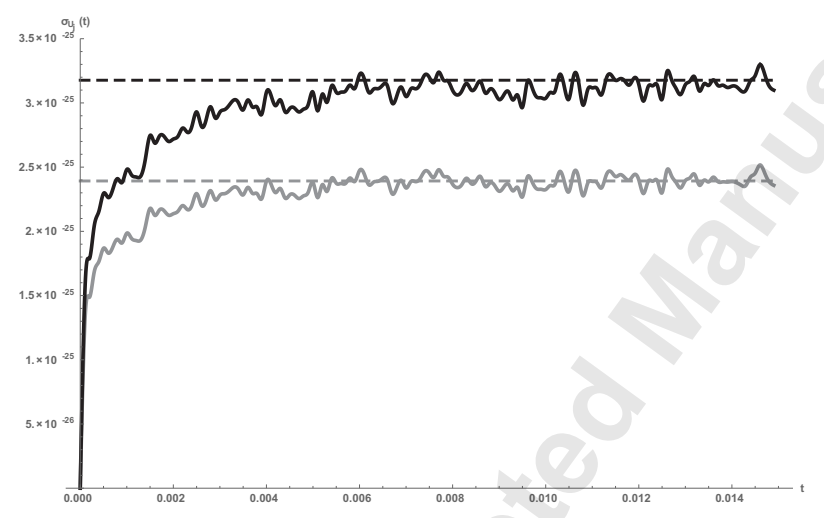

Figure 6. Displacement variance at $x=L$ (black) and $x=L / 2$ (gray) for VE case $\alpha=3 / 4$.

decreases.

\section{Concluding remarks}

The noisy non-local bar with fractional order term is a practical example of real mechanical problem in which the classical modal transformation fails to decouple the system of fractional differential equations that rules the motion. For this reason in this paper a method to decouple the equations of motion when fractional order terms appear has been intro-

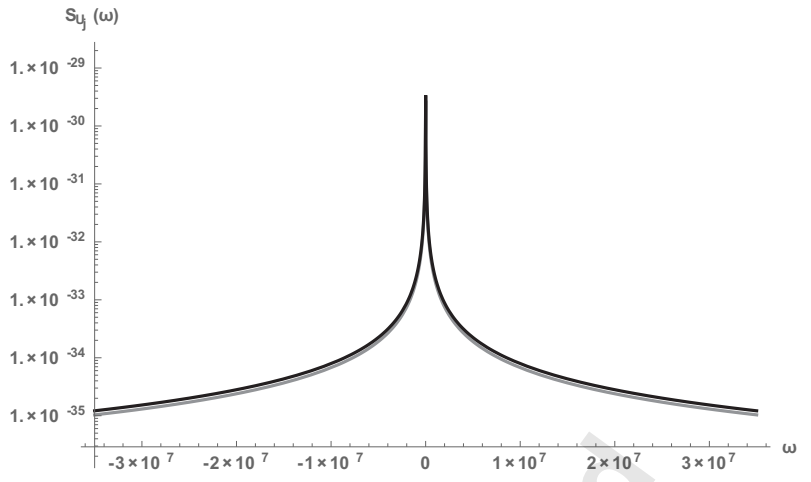

Figure 7. PSD of axial displacement at $x=L$ (black) and $x=L / 2$ (gray) for VE case $\alpha=3 / 4$.

duced.

The bar has been discretized with the FE formulation of the nonlocal beams provided by the authors in previous papers which closed form matrices are available. Once the FE model of the bar has been constructed it can be treated as a generic fractional multi-degree of freedom system. The described state variable expansion and the complex modal transformation have allowed to integrate the solution in the time domain and also to perform Monte Carlo simulation very efficiently.

The study has been focused to the evaluation of the effect of the fractional order on the statistics of the response. In particular, the influence of the fractional order on the PSD response has been investigated. The numerical results, corroborated by the Monte Carlo simulation, have been shown that the increasing of the fractional order leads to a reduction of the amplitude of the PSD function.

\section{References}

[1] Lakes, R.S., 1991. "Experimental micro mechanics methods for conventional and negative Poisson's ratio cellular solids as Cosserat continua". Journal of Engineering Materials and Technology, 113(1), pp. $148-155$.

[2] Aifantis, E.C., 1994. "Gradient effects at macro, micro, and nano scales". Journal of Mechanical Behaviour of Materials, 5(3), pp. 355-375.

[3] Qian, D., Wagner, G.J., Liu, W.K., Yu, M.-F., and Ruoff, R.S., 2002. "Mechanics of carbon nanotubes". Applied Mechanics Review, 55(6), pp. 495-533.

[4] Arash, B., and Wang, Q. 2012. "A review on the application of nonlocal elastic models in modeling of carbon nanotubes and graphenes". Computational Materials Science, 51(1), pp. 303-313.

[5] Wang, L. F., and Hu, H. Y., (2005). "Flexural wave propagation in single-walled carbon nanotube." Physical Review B, 71(19), pp. 195412-195418.

[6] Tang, P.Y., 1983. "Interpretation of bend strength increase of graphite by the couple stress theory". Computers and Structures, 16(1-4), pp. 45-49.

[7] Poole, W.J., Ashby, M.F., and Fleck, N.A., 1996. 
Copyright (c) 20، Micro-hardness of annealed and work-hardened copper polycrystals". Scripta Materialia, 34(4), pp. 559-564.

[8] Lam, D.C.C., Yang, F., Chong, A.C.M., Wang, J., and Tong, P., 2003. "Experiments and theory in strain gradient elasticity". Journal of Mechanics and Physics of Solids, 51(8), pp. 1477-1508.

[9] McFarland, A.W., and Colton, J.S., 2005. "Role of material microstructure in plate stiffness with relevance to microcantilever sensors". Journal of Micromechanics and Microengineering, 15(5), pp. 1060-1067.

[10] Russell, D.L., 1992. "On mathematical models for the elastic beam with frequency-proportional damping". Control and estimation in distributed parameter systems, H. T. Banks, ed., SIAM, Philadelphia, pp. 125-169.

[11] Banks, H.T., and Inman, D.J., 1991. "On damping mechanism in beams". Journal of Applied Mechanics, 58(3), pp. 716-723.

[12] Lei, Y., Friswell, M.I., and Adhikari, S., 2006. "A Galerkin method for distributed systems with non-local damping". International Journal of Solids and Structures, 43(11-12), pp. 3381-3400.

[13] Friswell, M.I., Adhikari, S., and Lei, Y., 2007. "Nonlocal finite element analysis of damped beams". International Journal of Solids and Structures, 44(22-23), pp. 7564-7576.

[14] Eringen, A.C., 1972. "Linear theory of non-local elasticity and dispersion of plane waves". International Journal Engineering Science, 10(5), pp. 425-435.

[15] Eringen, A.C., 1983. "On differential equations of nonlocal elasticity and solutions of screw dislocation and surface waves." Journal of Applied Physics, 54(9), pp. 4703-4710.

[16] Lu, P., Lee, H.P., Lu, C., and Zhang, P.Q., 2007. "Application of nonlocal beam models for carbon nanotubes". International Journal of Solids and Structures, 44(16), pp. 5289-5300.

[17] Pradhan, S.C., Phadikar, J.K., 2009. "Nonlocal elasticity theory for vibration of nanoplates". Journal of Sound and Vibration, 325(1-2), pp. 206-223.

[18] Park, S.K., and Gao, X.L., 2006. "Bernoulli-Euler beam model based on a modified couple stress theory". Journal of Micromechanics and Microengineering, 16(11), pp. 2355-2359.

[19] Kong, S., Zhou, S., Nie, Z., and Wang, K., 2008. "The size-dependent natural frequency of BernoulliEuler micro-beams". International Journal Engineering Science, 46(5), pp. 427-437.

[20] Ma, H.M., Gao, X.-L., and Reddy, J. N., 2008. "A microstructure dependent Timoshenko beam model based on a modified couple stress theory." Journal of Mechanics and Physics of Solids, 56(12), pp. 3379-3391.

[21] Challamel, N., and Wang, C.M., 2008. "The small length scale effect for a non-local canti-lever beam: A paradox solved". Nanotechnology, 19(34), pp. 345703.

[22] Zhang, Y.Y., Wang, C.M., and Challamel, N., 2010. "Bending, buckling, and vibration of micro/nanobeams by hybrid nonlocal beam model". Journal of Engineering Mechanics, 10.1061/(ASCE)EM.19437889.0000107, pp. 562-574.

[23] Payton, D., Picco, L., Miles, M.J., Homer, M.E., and Champneys, A.R., 2012. "Modelling oscillatory flexure modes of an atomic force microscope cantilever in contact mode whilst imaging at high speed". Nanotechnology, 23(26), pp. 265702.

[24] Murmu, T., and Adhikari, S., 2012. "Nonlocal frequency analysis of nanoscale biosensors." Sensor and Actuators A-Physical, 173(1), pp. 41-48.

[25] Chen, C., Ma, M., Liu, J., Zheng, Q., and Xu, Z., 2011. "Viscous damping of nanobeam resonators: Humidity, thermal noise, and a paddling effect." Journal of Applied Physics, 110(3), pp. 034320.

[26] Lee, J., and Lin, C., 2010. "The magnetic viscous damping effect on the natural frequency of a beam plate subject to an in-plane magnetic field". Journal of Applied Mechanics, 77(1), pp. 011014.

[27] Di Paola, M., Failla, G., and Zingales, M., 2013. "Non-local stiffness and damping models for sheardeformable beams". European Journal of Mechanics A/Solids, 40, pp. 69-83.

[28] Di Paola, M., Failla, G., and Zingales, M., 2014. "Mechanically based nonlocal Euler-Bernoulli beam model". Journal of Micromechanics and Microengineering, 10.1061/(ASCE)NM.2153-5477.0000077, A4013002.

[29] Failla, G., Sofi, A., and Zingales, M., 2015. "A new displacement-based framework for non-local Timoshenko beams". Meccanica, 50(8), pp. 2103-2122.

[30] Alotta, G., Failla, G., and Zingales, M., 2014. "Finite element method for a nonlocal Timoshenko beam model". Finite Element in Analysis and Design, 89, pp. 77-92.

[31] Fuchs, M.B., 1991. "Unimodal beam elements". International Journal of Solids and Structures, 27(5), pp. 533-545.

[32] Fuchs, M.B., 1997. "Unimodal formulation of the analysis and design problems for framed structures". Computers and Structures, 63(4), pp. 739-747.

[33] Phadikar, J.K., Pradhan, S.C., 2010. "Variational formulation and finite element analysis for nonlocal elastic nanobeams and nanoplate". Computational Materials Science, 49(3), pp. 492-499.

[34] Pradhan, S.C. (2012). "Nonlocal finite element analysis and small scale effects of CNTs with Timoshenko beam theory." Finite Element in Analysis and Design, 50, pp. $8-20$.

[35] Alotta, G., Failla, G., and Zingales, M., 2015. "Finite-Element Formulation of a Nonlocal Hereditary Fractional-Order Timoshenko Beam". Journal of Engineering Mechaincs (ASCE), 10.1061/(ASCE)EM.1943-7889.0001035.

[36] Podlubny, I., 1999. Fractional differential equations: An introduction to fractional derivatives, fractional differential equations, some methods of their solution and some of their applications. Academic Press, New York. 

linear viscoelasticity: An introduction to mathematical models. World Scientific, Singapore.

[38] Alotta, G., Di Paola, M., Pirrotta, A., 2014. "Fractional Tajimi-Kanai model for simulating earthquake ground motion". Bulletin of Earthquake Engineering, 12, pp. 2495-2506.

[39] Di Paola, M., Fiore, V., Pinnola, F.P., and Valenza, A., 2014. "On the influence of the initial ramp for a correct definition of the parameters of fractional viscoelastic materials". Mechanics of Materials, 69(1), pp. 63-70.

[40] Bagley, R.L., and Torvik, P.J., 1983. "A theoretical basis for the application of fractional calculus to viscoelasticity”. Journal of Rheology, 27(3), pp. 201-210.

[41] Bagley, R.L., and Torvik, P.J., 1986. "On the fractional calculus model of viscoelastic behavior". Journal of Rheology, 30(1), pp. 133-155.

[42] Failla, G., and Pirrotta, A., 2012. "On the stochastic response of a fractionally-damped Duffing oscillator". Communication in Nonlinear Science and Numerical Simulations, 17(12), pp. 5131-5142.

[43] Bagley, R.L., and Torvik, P.J., 1985. "Fractional Calculus in the Transient Analysis of viscoelastically Damped Structures". AIAA Journal, 23(6), pp. 918925.

[44] Di Paola, M., Pinnola, F.P., and Spanos, P.D., 2014 "Analysis of multi-degree-of-freedom systems with fractional derivative elements of rational order". Proceedings of ICFDA14 (IEEE).

[45] Di Paola, M., Failla, G., and Zingales, M., 2010. "The mechanically based approach to 3D non-local linear elasticity theory: Long-range central interactions". International Journal of Solids and Structures, 47(18-19), pp. 2347-2358.

[46] Altan, B.S., 1989. "Uniqueness of the initialvalue problems in non-local elastic solids". International Journal of Solids and Structures, 25(11), pp. 1271-1278.

[47] Polizzotto, C., 2001. "Non local elasticity and related variational principles". International Journal of Solids and Structures, 38(42-43), pp. 7359-7380.

[48] Samko, S.G., Kilbas, A.A., Marichev, O.I., 1993.Fractional integral and derivatives. Theory and applications. Gordon and Breach Science Publishers.

[49] Di Paola, M., Failla, G., and Zingales, M., 2009. "Physically-based approach to the mechanics of strong non-local linear elasticity theory". Journal of Elasticity, 97(2), pp. 103-130.

[50] Reddy, J.N., 1999. "On the dynamic behaviour of the Timoshenko beam finite elements". Sādhanā, 24(3), pp. 175-198.

[51] Pinnola, F.P., 2016. "Statistical correlation of fractional oscillator response by complex spectral moments and state variable expansion". Communications in Nonlinear Science and Numerical Simulation, 39, pp. 343359.

[52] Shinozuka, M., Deodatis, G. 1988. "Stochastic process models for earthquake ground motion". Probabilistic 